Original Article

\title{
Comparison of abdominal muscle activity and peak expiratory flow between forced vital capacity and fast expiration exercise
}

\author{
Hiroshi Ishida, RPT, PhD ${ }^{1)^{*}}$, TAdanobu Suehiro, RPT, PhD $^{1)}$, Susumu Watanabe, RPT, PhD ${ }^{1)}$ \\ 1) Department of Rehabilitation, Faculty of Health Science and Technology, Kawasaki University of \\ Medical Welfare: 288 Matsushima, Kurashiki, Okayama 701-0193, Japan
}

\begin{abstract}
Purpose] The purpose of this investigation was to compare the activities of the abdominal muscles and peak expiratory flow between forced vital capacity and fast expiration exercise. [Subjects and Methods] Fifteen healthy male participated in this study. Peak expiratory flow and electromyographic activities of the rectus abdominis, external oblique, and internal oblique/transversus abdominis muscles were measured during forced vital capacity and fast expiration exercise and then peak amplitude and its appearance time were obtained. [Results] Peak expiratory flow values were significantly higher during fast expiration exercise than during forced vital capacity. The internal oblique/transversus abdominis muscles showed significantly higher peak amplitude during fast expiration exercise than during forced vital capacity. However, there were no significant differences between forced vital capacity and fast expiration exercise in the rectus abdominis and external oblique muscles. There was no difference in the appearance time of the peak amplitude between forced vital capacity and fast expiration exercise in any muscle. [Conclusion] Fast expiration exercise might be beneficial for increasing expiratory speed and neuromuscular activation of the internal oblique/transversus abdominis muscles compared to forced vital capacity. These findings could be considered when recommending a variation of expiratory muscle strength training as part of pulmonary rehabilitation programs.

Key words: Expiratory muscle strength training, Electromyography, Abdominal muscles
\end{abstract}

(This article was submitted Oct. 20, 2016, and was accepted Dec. 15, 2016)

\section{INTRODUCTION}

Surpassing stroke, pneumonia is the third leading cause of death in Japan, and deaths from pneumonia are more prevalent in elderly individuals ${ }^{1)}$. The pathophysiology of pneumonia in the elderly is primarily aspiration pneumonia ${ }^{2}$. Aspiration pneumonia is associated with reduced swallow and cough capacity ${ }^{3)}$. Expiratory muscle strength training (EMST) programs are known to increase maximum expiratory pressure, with the potential to improve some aspects of swallow, cough, and physical performance ${ }^{4)}$.

In general, EMST performed using pressure threshold devices strengthens the expiratory muscles by increasing the expiratory load during breathing exercises ${ }^{4}$. Recently, we showed that 4 weeks of fast expiration (FE) exercises performed without pressure improved respiratory muscle strength ${ }^{5}$. During FE exercise, the investigator instructed the subjects to inspire fully to total lung capacity, to blow through a mouthpiece as fast as possible, to concentrate on the speed of instantaneous expiration and not on the volume, and to not exhale to residual volume ${ }^{5)}$. Generally, verbal instruction for pulmonary function measurements of forced vital capacity (FVC) is to exhale from total lung capacity to residual volume as fast as possible. Whether instantaneous expiration during FE exercise is an effective method to activate expiratory muscles is unclear. If the

*Corresponding author. Hiroshi Ishida (E-mail: ishida@mw.kawasaki-m.ac.jp)

(C)2017 The Society of Physical Therapy Science. Published by IPEC Inc.

This is an open-access article distributed under the terms of the Creative Commons Attribution Non-Commercial No Derivatives (by-nc-nd) License $<$ https://creativecommons.org/licenses/by-nc-nd/4.0/>. 
motor units are activated in a task-specific manner, expiratory muscle activity will differ with the task. The amplitude of the agonist burst is proportional to the peak velocity when the load is constant ${ }^{6}$. The abdominal muscles are the muscles of forced expiration ${ }^{7}$. Expiratory muscle strength reflects peak expiratory flow (PEF) in the absence of bronchial obstruction ${ }^{8)}$. Therefore, the purpose of this investigation was to compare the activities of the abdominal muscles and PEF between FVC and FE exercise.

\section{SUBJECTS AND METHODS}

Fifteen healthy male university students participated in this trial. The subjects were physiotherapy students attending the Kawasaki University of Medical Welfare. The protocol for the present study was approved by the Ethics Committee of the Kawasaki University of Medical Welfare. Written informed consent was obtained and the rights of the subjects were protected. Subjects with a history of chronic or acute cardiac, pulmonary, or neuromuscular disease or smoking, and those who had an acute upper respiratory infection were excluded. All pulmonary function measurements, which included percentage of the predicted vital capacity (\%VC: vital capacity/predicted vital capacity) and forced expiratory volume in one second $\left(\mathrm{FEV}_{1} \%\right.$ : forced expiratory volume in one second/FVC), were calculated using a multi-functional spirometer (HI-801; Chest MI Inc., Tokyo, Japan). All the measurement values were within the normal range.

Disposable silver/silver chloride surface electrodes with a recording diameter of $1 \mathrm{~cm}$ (Blue Sensor P-00S; Mets Co. Ltd., Tokyo, Japan) were used. The electromyographic (EMG) signals were recorded using a data acquisition system (Myosystem 1200; Noraxon Inc., AZ, USA). Electrode placement was based on previous work ${ }^{9,10)}$ that noted the muscle positions on the right side as follows: the rectus abdominis (RA), $2.5 \mathrm{~cm}$ lateral to the umbilicus; external oblique (EO), the anterior end of the eighth rib; internal oblique and transversus abdominis ( $\mathrm{IO} / \mathrm{TrA})$, midpoint between the anterior-superior iliac spine and pubic symphysis. Skin preparation gel (Skin Pure; Nihon Kohden Co. Ltd., Tokyo, Japan) was applied to the skin before electrode placement; subsequently, the skin was cleaned with alcohol to reduce skin surface impedance. Bipolar electrode pairs were placed longitudinally over the muscle at $2.5-\mathrm{cm}$ intervals. A grounded electrode was placed over the anterior superior iliac spine.

PEF (L/min) was measured using a peak flow meter (Assess, Full range; Philips Respironics G.K. Tokyo, Japan) with a mouthpiece. To detect onset of expiratory flow, temperature change $1 \mathrm{~cm}$ distal to the mouth side of the mouthpiece was measured using the thermistor (TR861-T; Nihon Kohden Co. Ltd., Tokyo, Japan). Electric signals from the thermistor were recorded synchronously with the EMG data using the external input board (BNC EM-135; Noraxon Inc., AZ, USA). The EMG and thermistor signals were amplified, band-pass filtered $(10-500 \mathrm{~Hz})$, digitized, and stored with a sample frequency of $1,000 \mathrm{~Hz}$.

The subjects were placed in the standing position. EMG and thermistor signals were recorded during FVC and FE exercise. Verbal instructions for FVC tasks were as follows 5): "inspire fully to total lung capacity, and then blow through a mouthpiece as fast as possible to residual volume." The verbal instructions for FE exercise were as follows: "inspire fully to total lung capacity and then blow through a mouthpiece as fast as possible, concentrate not on the volume but on the speed of instantaneous expiration, and it is not necessary to exhale to residual volume." Subjects were allowed to practice until they could perform the tasks consistently. Data were collected 3 times for each task. The order of measurements for the 2 tasks was randomly assigned. The onset of expiratory flow was decided visually at the time of a sharp change of thermistor signals using MyoResearch software (Noraxon Inc., AZ, USA). Peak amplitude and its relative appearance time were detected using BIMUTASII software (Kissei Comtec Co., Ltd., Nagano, Japan). The pre 200 msec EMG signals of the expiratory onset time were loaded. Then, EMG signals were full-wave rectified, and the peak amplitudes $(\mathrm{mV})$ were determined. The relative appearance time of peak amplitude was calculated by the following equation: appearance time = peak amplitude appearance of each muscle - expiratory flow onset (msec).

PEF, peak amplitude, and appearance time data in the 3 trials performed for each tasks were then averaged and used in statistical analysis. SPSS Statistics 23.0 (SPSS Inc., Chicago, IL, USA) was used for the statistical analysis. Differences between FVC and FE exercise were analyzed using the paired t-test. Values were considered statistically significant at values of $p<0.05$.

\section{RESULTS}

The characteristics of the subjects are shown in Table 1. PEF values were significantly higher during FE exercise than during FVC (Table 2). The IO/TrA muscles showed significantly higher peak amplitude during FE exercise than during FVC. There were no significant differences between FVC and FE exercise in the RA and EO muscles. There was no difference in the appearance time of the peak amplitude between FVC and FE exercise in any muscle.

\section{DISCUSSION}

To the best of our knowledge, this is the first study to compare abdominal muscle activity and expiratory speed between FVC and FE exercise. In this study, PEF was higher during FE exercise than during FVC. Variations in the speed, amplitude, 
Table 1. Characteristics of the subject

\begin{tabular}{lc}
\hline & Characteristics \\
\hline Age (years) & $20.9 \pm 0.5$ \\
Height $(\mathrm{cm})$ & $169.6 \pm 3.8$ \\
Weight $(\mathrm{kg})$ & $60.6 \pm 9.0$ \\
$\% \mathrm{VC}$ & $102.9 \pm 13.8$ \\
$\mathrm{FEV}_{1} \%$ & $90.4 \pm 4.3$ \\
\hline$\% \mathrm{VC}_{\text {: vital capacity/predicted vital ca- }}$ \\
pacity; FEV ${ }_{1}$ : forced expiratory volume \\
in one second/forced vital capacity
\end{tabular}

Table 2. Mean \pm standard deviation of the PEF, peak amplitude, and appearance time

\begin{tabular}{llcc}
\hline & & FVC & FE exercise \\
\hline PEF (1/min) & & $536.4 \pm 87.4$ & $572.9 \pm 80.9^{*}$ \\
Peak amplitude & RA & $0.16 \pm 0.19$ & $0.17 \pm 0.11$ \\
(mV) & EO & $0.44 \pm 0.29$ & $0.46 \pm 0.24$ \\
& IO/TrA & $1.44 \pm 0.88$ & $1.58 \pm 0.84^{*}$ \\
Appearance & RA & $-29.4 \pm 13.0$ & $-28.5 \pm 11.5$ \\
time (msec) & EO & $-24.1 \pm 11.0$ & $-25.1 \pm 13.3$ \\
& IO/TrA & $-31.9 \pm 15.1$ & $-33.9 \pm 13.6$ \\
\hline
\end{tabular}

PEF: peak expiratory flow; FVC: forced vital capacity; FE: fast expiration; RA: rectus abdominis; EO: external oblique; IO/TrA: internal oblique and transversus abdominis $* \mathrm{p}<0.05$

or force of a movement can be produced by alterations in the motor program ${ }^{11,12)}$. In this study, the subjects needed to exhale as fast as possible to residual volume during FVC, so that the expiratory motor program was tasked to act strong for long. On the other hand, the subjects needed to concentrate on the speed of instantaneous expiration during FE exercise, so that the expiratory motor program was tasked only to act strong quickly. Literature proposed organizing principles for the control of single-joint human movements in which tasks are performed by one of two strategies, called speed-insensitive and speedsensitive strategies ${ }^{11,12}$. A model has been described, in which the movements made under a speed-insensitive strategy are executed by controlling the duration and relative timing of amplitude invariant patterns of activation to the spinal motor neuron pools ${ }^{11)}$. Another model has been proposed in which the movements made under a speed-sensitive strategy are executed by controlling the intensity of an excitation pulse delivered to the motor neuron pool ${ }^{12)}$. In this study, FE exercise produced higher activity in the IO/TrA muscle than the FVC, whereas appearance timing of the peak amplitude was not different between the two exercises. Therefore, movement under a speed-sensitive strategy might be made during the tasks, and different $\mathrm{PEF}$ values were recorded ${ }^{11,12)}$. In the literature, effects of explosive jump training and heavy resistance weight training on the force-time characteristics of isometric contractions of the knee extensors were compared ${ }^{13)}$. Explosive training caused a relatively greater increase in maximum rate of force development than in peak force, whereas heavy weight training caused a larger increase in peak force and a slight change in maximum rate of force development ${ }^{13)}$. If one of the goals of EMST is to develop expiratory speed, FE exercise should be included in the programs to consider a task-specific program. However, this study was not a prospective study. Further study is needed to compare treatment effect on expiratory muscle strength between FVC and FE maneuvers.In this study, the interesting finding was that, only the IO/TrA muscle activity was different between tasks, whereas there was no significant difference in RA and EO muscle activity. The proportion of Type I and Type II fibers was about equal in all the abdominal muscles, and a nearly equal proportion of IIA and IIB fibers were seen in the RA as well as in the EO and IO; however, TrA had a higher proportion of Type IIB fibers ${ }^{14)}$. On the basis of the histochemical analysis of muscle fiber composition, the TrA muscle might have advantage of a higher muscle contraction speed than the other abdominal muscles. Accordingly, the anatomical contribution of the $\mathrm{IO} / \mathrm{TrA}$ muscle to expiration is different from that of the RA and EO. The action of the RA produces a caudal displacement and decrease in the anteroposterior diameter of the rib cage, whereas the action of the EO causes a caudal displacement and decrease in the transverse diameter of the rib cage $\mathrm{e}^{15)}$. The activities of the TrA and IO pull the abdominal wall inward and increase intra-abdominal pressure ${ }^{16)}$. As the abdominal contents are virtually incompressible, the diaphragm moves cranially into the thoracic cavity ${ }^{16}$. This displacement results in an increase in pleural pressure and a decrease in lung volume ${ }^{16}$. The action on the diaphragm might have high compliance with the pleural pressure compared to the action on the rib cage owing to the elasticity of the tissue. Therefore, increase of $\mathrm{IO} /$ TrA muscle activity was selected to perform instantaneous expiration during FE exercise.

The present study has some limitations. The sample size is small. Another limitation of this study was that only young male subjects were recruited, so the influence of gender was not observed. As part of the normative aging process, there is an overall decline in skeletal muscle mass and strength. This decline has also been documented for the muscles of respiration ${ }^{17)}$. Further study including a larger sample and other age groups is needed to establish effective breathing maneuvers in the elderly.

Our results indicated that FE exercise might be beneficial for increasing expiratory speed and neuromuscular activation of the IO/TrA muscles compared to FVC. These findings study should be considered when recommending a variation of the EMST as part of a pulmonary rehabilitation program. 


\section{ACKNOWLEDGEMENTS}

This work was supported by JSPS KAKENHI Grant Number 15K16383. The authors wish to thank the subjects for their invaluable contribution to this study.

\section{REFERENCES}

1) Ministry of Health, Labor and Welfare in Japan: Vital Statistics, 2013. http://www.mhlw.go.jp/toukei/saikin/hw/jinkou/geppo/nengai14/d1/gaikyou26.pdf\#sea

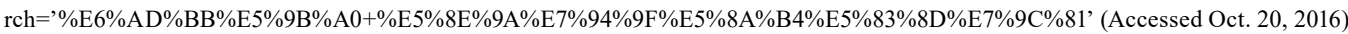

2) Teramoto S, Yoshida K, Hizawa N: Update on the pathogenesis and management of pneumonia in the elderly-roles of aspiration pneumonia. Respir Investig, 2015, 53: 178-184. [Medline] [CrossRef]

3) Ebihara S, Sekiya H, Miyagi M, et al.: Dysphagia, dystussia, and aspiration pneumonia in elderly people. J Thorac Dis, 2016, 8: 632-639. [Medline] [CrossRef]

4) Laciuga H, Rosenbek JC, Davenport PW, et al.: Functional outcomes associated with expiratory muscle strength training: narrative review. J Rehabil Res Dev, 2014, 51: 535-546. [Medline] [CrossRef]

5) Ishida H, Kuramoto Y, Ikeda D, et al.: Effects of fast expiration exercises without pressure on the respiratory muscle strength of healthy subjects. J Phys Ther Sci, 2016, 28: 2759-2762. [Medline] [CrossRef]

6) Mustard BE, Lee RG: Relationship between EMG patterns and kinematic properties for flexion movements at the human wrist. Exp Brain Res, 1987, 66: 247-256. [Medline] [CrossRef]

7) Neumann DA: Kinesiology of the musculoskeletal system. St. Louis: Elsevier, 2010.

8) Suárez AA, Pessolano FA, Monteiro SG, et al.: Peak flow and peak cough flow in the evaluation of expiratory muscle weakness and bulbar impairment in patients with neuromuscular disease. Am J Phys Med Rehabil, 2002, 81: 506-511. [Medline] [CrossRef]

9) McGill SM, Norman RW, Sharratt MT: The effect of an abdominal belt on trunk muscle activity and intra-abdominal pressure during squat lifts. Ergonomics, 1990, 33: 147-160. [Medline] [CrossRef]

10) Richardson CA, Jull G, Hodges P, et al.: Therapeutic exercise for spinal segmental stabilization in low back pain: scientific basis and clinical approach. London: Churchill Livingstone, 1999.

11) Gottlieb GL, Corcos DM, Agarwal GC: Organizing principles for single-joint movements. I. A speed-insensitive strategy. J Neurophysiol, 1989,62 : $342-357$. [Medline]

12) Corcos DM, Gottlieb GL, Agarwal GC: Organizing principles for single-joint movements. II. A speed-sensitive strategy. J Neurophysiol, 1989, 62: 358-368. [Medline]

13) Sale DG: Neural adaptation to resistance training. Med Sci Sports Exerc, 1988, 20: S135-S145. [Medline] [CrossRef]

14) Häggmark T, Thorstensson A: Fibre types in human abdominal muscles. Acta Physiol Scand, 1979, 107: 319-325. [Medline] [CrossRef]

15) Mier A, Brophy C, Estenne M, et al.: Action of abdominal muscles on rib cage in humans. J Appl Physiol 1985, 1985, 58: 1438-1443. [Medline]

16) De Troyer A, Estenne M: Functional anatomy of the respiratory muscles. Clin Chest Med, 1988, 9: 175-193. [Medline]

17) Chen HI, Kuo CS: Relationship between respiratory muscle function and age, sex, and other factors. J Appl Physiol 1985, 1989, 66: 943-948. [Medline] 